\begin{tabular}{|c|c|c|}
\hline \multirow{3}{*}{$\begin{array}{r}\text { Case Reports in } \\
\text { Gastroenterology }\end{array}$} & \multirow{2}{*}{\multicolumn{2}{|c|}{ Case Rep Gastroenterol 2014;8:381-386 }} \\
\hline & & \\
\hline & $\begin{array}{l}\text { DOI: 10.1159/000369964 } \\
\text { Published online: December 5, } 2014\end{array}$ & $\begin{array}{l}\text { ○ } 2014 \text { S. Karger AG, Basel } \\
\text { 1662-0631/14/0083-0381 } \$ 39.50 / 0 \\
\text { www.karger.com/crg }\end{array}$ \\
\hline & \multicolumn{2}{|c|}{$\begin{array}{l}\text { This is an Open Access article licensed under the terms of the Creative Commons } \\
\text { Attribution-NonCommercial } 3.0 \text { Unported license (CC BY-NC) (www.karger.com/OA } \\
\text { license), applicable to the online version of the article only. Distribution permitted for non } \\
\text { commercial purposes only. }\end{array}$} \\
\hline
\end{tabular}

\title{
Gastrostomy Intraperitoneal Bumper Migration in a Three-Year-Old Child: A Rare Complication following Gastrostomy Tube Replacement
}

\author{
Riccardo Guanà $^{a} \quad$ Luca Lonati $^{a} \quad$ Claudio Barletti $^{\mathrm{b}} \quad$ Fabio Cisarò $^{\mathrm{b}}$ \\ Ilaria Casorzoc Giulia Carbonaro ${ }^{\mathrm{a}}$ Antonella Lezo $^{\mathrm{d}}$ \\ Angelo Giovanni Delmonaco ${ }^{e} \quad$ Alessandro Mussa $^{\mathrm{e}}$ Martina Capitanio $^{a}$ \\ Davide Cussa $^{a}$ Riccardo Lemini $^{a}$ Jürgen Schleef ${ }^{a}$ \\ ${ }^{a}$ Division of Pediatric General Surgery, ${ }^{b}$ Division of Pediatric Gastroenterology, \\ 'Division of Pediatric Radiology, ${ }^{\mathrm{d}}$ Division of Nutrition and ${ }^{\mathrm{e}}$ Department of Pediatrics, \\ Regina Margherita Children's Hospital, Turin, Italy
}

\section{Key Words}

Bumper migration · Gastrostomy · Gastrostomy tube replacement

\begin{abstract}
Feeding gastrostomy is used worldwide for adults and children with feeding impairment to obtain long-term enteral nutrition. Percutaneous endoscopic gastrostomy insertion is considered the gold standard, but after the first months requires gastrostomy tube replacement with a low-profile button. The replacement is known as an easy procedure, but several minor and major complications may occur during and after the manoeuvre. We describe intraperitoneal bumper migration in a 3-year-old boy, a rare complication following gastrostomy tube replacement, and we discuss the recent literature regarding similar cases.
\end{abstract}

(C) 2014 S. Karger AG, Basel

\section{Introduction}

A gastrostomy is the creation of an artificial external opening into the stomach for nutritional support. The procedure consists in creating a fistula tract that connects the stomach to the abdominal wall to get a direct access to the gastric lumen. Several clinical conditions exist in which, despite preserved digestive function, it is not possible to reach adequate oral

Riccardo Guanà, MD

Regina Margherita Children's Hospital

Piazza Polonia 94

IT-101026 Turin (Italy)

E-Mail riccardoguan@gmail.com 
Guanà et al.: Gastrostomy Intraperitoneal Bumper Migration in a Three-Year-Old Child: A Rare Complication following Gastrostomy Tube Replacement

feeding, with the onset of malnutrition. If anatomical obstacles exist to the positioning of a nasogastric tube or if the expected period of necessary enteral nutrition is more than 6 weeks, the use of a nasogastric tube is usually not recommended, and in these cases the creation of a gastrostomy is the only alternative.

The majority of patients who require a gastrostomy are subjects with neurological diseases. These patients often present neuromuscular incoordination that compromises the regular sequence of swallowing and leads to gastro-oesophageal reflux disease, responsible of repeated episodes of inhalation and chronic respiratory disease, which make obtaining a normal diet impossible.

The percutaneous endoscopic gastrostomy (PEG) technique was initially proposed in the paediatric age group by Gauderer and Ponsky in 1980 [1]. Since then, it has become the technique of choice for gastrostomy worldwide where there are no anatomical or anaesthesiological contraindications to its execution. The procedure consists in introducing a gastroscope, inflating and distending the stomach, and identifying the correct location where to place the gastrostomy. Finger pressure is applied at the abdominal point of maximal transillumination, and a focal indentation of the anterior gastric wall is visible endoscopically. Then, puncture of the abdominal wall with a needle catheter is achieved under endoscopic control right in the stomach. After removing the needle, and leaving the catheter in place, a guide-wire is introduced into the stomach, which is grasped by an endoscopic loop introduced through the gastroscope channel and carried out outside of the mouth. The end of the gastrostomy tube is secured to the guide-wire, which is withdrawn in the stomach so as to allow the gastrostomy tube to reach the gastric lumen. Continuing the traction, the probe is then brought outside the abdominal wall, starting from its rigid tail, until the opposite end of the probe, consisting of a terminal bumper, so that it cannot exit the small foramen, and at the same time pulls and maintains approached the gastric wall to the abdominal wall. At the end of the procedure the probe is left open so as to decompress the stomach and can be usually used for feeding after $24 \mathrm{~h}$. It is also possible to perform a socalled 'push' gastrostomy where, after placing the guide-wire exactly as in the 'pull' technique, the gastrostomy tube is advanced through the abdominal wall along the guide-wire itself, driven by an appropriate introducer.

The fibrous adhesions which occur between the stomach wall and the abdominal wall allow to obtain a spontaneous gastropexy after about 3 months, and after that period the 'primitive' gastrostomy tube can usually be safely replaced with a 'low-profile' tube. The PEG tube should be removed by either gently pulling it through the stoma (if the tube diameter is $12 \mathrm{Fr}$ ) or through endoscopic retrieval (if the tube is $14 \mathrm{Fr}$ or larger). It is not recommended to cut a portion of the tube in order to allow the internal bumper to pass; nevertheless, also in case of 12-Fr tube, if the device cannot be removed with a reasonable amount of traction, it should be removed by endoscopic retrieval.

Complications following gastrostomy tube replacement are well known and include fistula disruption, colocutaneous fistula, pancreatitis, cholangitis, haemorrhage, gastric ulcer as well as oesophageal or duodenal perforation [2]. Moreover, a gastrostomy internal bumper can migrate through the pylorus and into the small bowel and colon, producing gastric outlet or small bowel or colonic obstruction. Using low-profile gastrostomy tubes is a way to reduce ballooning-associated complications, but other complications, such as buried bumper syndrome and gastric ulcer, might be encountered.

We describe an unusual case of intraperitoneal bumper migration following gastrostomy tube replacement. 
Guanà et al.: Gastrostomy Intraperitoneal Bumper Migration in a Three-Year-Old Child: A Rare Complication following Gastrostomy Tube Replacement

\section{Case Presentation}

The patient was born on September 6, 2011, suffering by the age of 1 year from severe anorexia from emotional deprivation that was progressively getting worse over time, with the complete impossibility of oral feeding. He was initially studied from a neurological point of view in another centre, and after a period of enteral feeding with a nasogastric tube, on November 2012, an indication for PEG was made (gastrostomy tube 14 Fr; Bard Access Systems Inc., Covington, Ga., USA). No problems were encountered during the procedure and using the gastrostomy tube for the first 3 months.

In the fourth month, on December 3, 2013, it was decided to replace the gastrostomy tube, and given the impossibility to extract the bumper by simple traction, the latter was cut and left in the gastric cavity, inserting the new probe (Kangaroo gastrostomy feeding tube, Covidien, Mansfield, Mass., USA) and inflating the balloon with water to check proper functioning. An immediate endoscopic control was prudently done. During the gastroscopy, the bumper was not visualized in the stomach and was supposed to have already migrated into the duodenum. After $24 \mathrm{~h}$ the patient presented fever, slight abdominal pain and secretions from the peristomal skin.

On December 5, 2013 an abdominal X-ray was obtained, evidencing the bumper in the descending colon. The family of the boy was reassured with the certainty of bumper emission in a short time and the patient was discharged home. Fourth months later, in April 2014, the clinical practitioner sent the boy to the emergency department (ED) for failure of bumper expulsion with the stool. An abdominal X-ray was requested and the bumper was again reported in the sigmoid colon (fig. 1). In June a barium enema allowed to hypothesize a radiopaque foreign body present in left pelvic region; the following month it was decided to submit the patient to total colonoscopy under sedation but, during the procedure, no bumper was found. In August a third abdominal X-ray reconfirmed a foreign body in the left pelvic region. During the following days the patient was hospitalized in our service and we decided to perform laparoscopic exploration of the abdomen under the suspicion of ileal retention.

Surprisingly, at the operation (performed with one-trocar laparoscopy with a 0 -degree laparoscope equipped with an operative channel) the bumper was visualized in the left iliac fossa, outside the intestine, enveloped by adhesions. Simple adhesiolysis with an atraumatic grasper allowed to mobilize the bumper and to extract it from the navel (fig. 2). The patient was discharged the next day and the follow-up period was uneventful.

\section{Discussion}

Gastrostomy tube replacement is known as an easy and safe procedure, but various complications are reported in literature; the overall reported complication rate is $1.3 \%$, and fistula disruption is the most common complication and known as a critical emergency that lead to peritonitis.

In most of cases, if the complications are detected early and peritonitis does not develop, simple reposition of the gastrostomy tube and conservative care are sufficient in many cases. A confirmatory imaging study (contrast study via the replaced gastrostomy tube) after replacement may be helpful for early detection of this complication (duodenal dislocation), but instead of post-manoeuvre confirmatory images, tube replacement with radiologic guide, such as fluoroscopy, can be considered. Endoscopy-guided tube change may be the 
Guanà et al.: Gastrostomy Intraperitoneal Bumper Migration in a Three-Year-Old Child: A Rare Complication following Gastrostomy Tube Replacement

safest way under the aspect of reducing balloon- and tube-associated complication, but patients should be sedated and may sometimes require general anaesthesia.

In our experience, waiting a minimum of 6 months after initial PEG before replacing the gastrostomy tube could allow for more stable fistula formation and avoid bumper dislocation. However, devices guidelines suggest not to remove a 14-Fr gastrostomy tube by traction, but to cut the gastric bumper under direct vision while performing gastroscopy and to immediately recuperate the internal bumper.

Exploring the recent literature, we found that Kim et al. [2] described duodenal perforation as an unusual and acute complication of gastrostomy tube replacement in a 2-year-old girl, underlining the risk of this manoeuvre in small children with small stomachs.

McSweeney et al. [3], in 2013, examined tube-related (positioning and replacement) major complications in paediatric patients undergoing PEG placement during a 2-year follow-up period in a retrospective chart review at Boston Children's Hospital. Among 138 patients, $11 \%$ had at least one major complication related to the gastrostomy tubes during the examined time period. The report concluded that children undergoing PEG placement have a long-term high risk of morbidity related to enteral tubes and that major complications can occur many years after PEG placement.

Brewster et al. [4], in 2012, prospectively followed 103 children undergoing PEG to establish a clear understanding of the complication rates associated with this procedure and with the use of the device over time. There were no intraoperative complications, with a $100 \%$ procedure completion rate, but the total complication rate was $14 \%$, although rates of PEG complications observed in this prospective study were low and are generally minor. The authors noted that the observed rates of PEG-specific complications were lower than in historic reports.

Fascetti-Leon et al. [5], in 2012, conducted a multi-centre prospective clinical data collection during 3 years in order to establish the mortality and morbidity of PEG in a large cohort of children; 239 children were enrolled. They stated that the presence of thoracoabdominal deformity was an independent predictor of complications at 12 months, but no risk factors were identified in association with complications during the first tube replacement. They concluded that in children undergoing PEG placement minor complications are common, while severe morbidities are rare. Accurate follow-up is essential to recognize every complication, in particular when risk factors such as thoraco-abdominal deformity exist.

Showalter et al. [6], in 2012, determined the frequency of misplacement and subsequent complications for children undergoing gastrostomy tube replacement in a paediatric ED and the impact of contrast-enhanced confirmatory imaging on ED length of stay. It was a retrospective review of children presenting to a paediatric ED over 16 months. A total of 237 children were enrolled; $1.2 \%$ had evidence of gastrostomy tube misplacement, all of whom underwent confirmatory imaging. One complication from misplacement was identified (gastric outlet obstruction from an overfilled balloon), and 35\% of patients had confirmatory imaging performed after replacement. The conclusion was that in the presence of clinical confirmation, confirmatory imaging may be judiciously used.

Peters et al. [7], in 2010, reviewed the outcome and complications of laparoscopicassisted gastrostomy insertion in a retrospective case note review, stating that PEG insertion is invariably associated with a risk of intestinal perforation and frequently requires a second anaesthesia for its replacement with a low-profile button. They claimed that the laparoscopic technique with low-pressure insufflation would yield comparable outcomes as well as a lower procedural complication rate and require fewer anaesthetics per patient. He studied 114 patients within a 8-year period, with $86 \%$ of children undergoing laparoscopic-assisted 
Guanà et al.: Gastrostomy Intraperitoneal Bumper Migration in a Three-Year-Old Child: A Rare Complication following Gastrostomy Tube Replacement

PEG. Children had their original gastrostomy changed to a button at a median interval of 7 months. Complications observed included new or deteriorating gastro-oesophageal reflux $(n=16)$, infection $(n=9)$, granulation tissue $(n=11)$, tube dislodgement $(n=2)$ and intraabdominal leakage of feed $(n=2)$. There were two gastrocolic fistulae which occurred in the standard PEG group. Inadvertent formation of a gastrocolic fistula was avoided with the use of laparoscopy to aid PEG insertion. Laparoscopic gastrostomy tube insertion was safe and easy to perform, with outcomes comparable to those of PEG tube insertion. It obviates the need for a second procedural anaesthetic and may emerge as the gold standard for gastrostomy tube placement.

Analysing our patient's history, we can state that two events occurred, opposite to each other. The first and the dramatic one was the gastrocutaneous fistula disruption due to the excessive and premature traction for removing the primitive PEG tube. The secondly and the lucky one was the immediate and correct fortuitous repositioning of the new tube into the gastric orifice which had just collapsed. Without this second manoeuvre the patient would probably have experienced progressive peritonitis and maybe necessity for abdominal surgical exploration and revision of the gastrostomy.

\section{Conclusion}

After gastrostomy tube replacement, when patients show irritability, fever with unknown origin and abdominal pain, fistula disruption must be considered. Safe replacement of the gastrostomy tube respecting international guidelines prevent further morbidity in patients who usually are already suffering from chronic diseases.

\section{References}

1 Gauderer MW, Ponsky JL, Izant RJ Jr: Gastrostomy without laparotomy: a percutaneous endoscopic technique. 1980. Nutrition 1998;14:736-738.

$\checkmark 2$ Kim SH, Min SH, Kim HY, Jung SE: Duodenal perforation: unusual complication of gastrostomy tube replacement. Pediatr Gastroenterol Hepatol Nutr 2014;17:112-115.

-3 McSweeney ME, Jiang H, Deutsch AJ, Atmadja M, Lightdale JR: Long-term outcomes of infants and children undergoing percutaneous endoscopy gastrostomy tube placement. J Pediatr Gastroenterol Nutr 2013;57: 663-667.

4 Brewster BD, Weil BR, Ladd AP: Prospective determination of percutaneous endoscopic gastrostomy complication rates in children: still a safe procedure. Surgery 2012;152:714-719; discussion 719-721.

$\checkmark 5$ Fascetti-Leon F, Gamba P, Dall'Oglio L, Pane A, dé Angelis GL, Bizzarri B, Fava G, Maestri L, Cheli M, Di Nardo G, La Riccia A, Marrello S, Gandullia P, Romano C, D’Antiga L, Betalli P: Complications of percutaneous endoscopic gastrostomy in children: results of an Italian multicenter observational study. Dig Liver Dis 2012;44:655-659.

-6 Showalter CD, Kerrey B, Spellman-Kennebeck S, Timm N: Gastrostomy tube replacement in a pediatric ED: frequency of complications and impact of confirmatory imaging. Am J Emerg Med 2012;30:1501-1506.

7 Peters RT, Balduyck B, Nour S: Gastrostomy complications in infants and children: a comparative study. Pediatr Surg Int 2010;26:707-709. 


\section{Case Reports in \\ Gastroenterology}
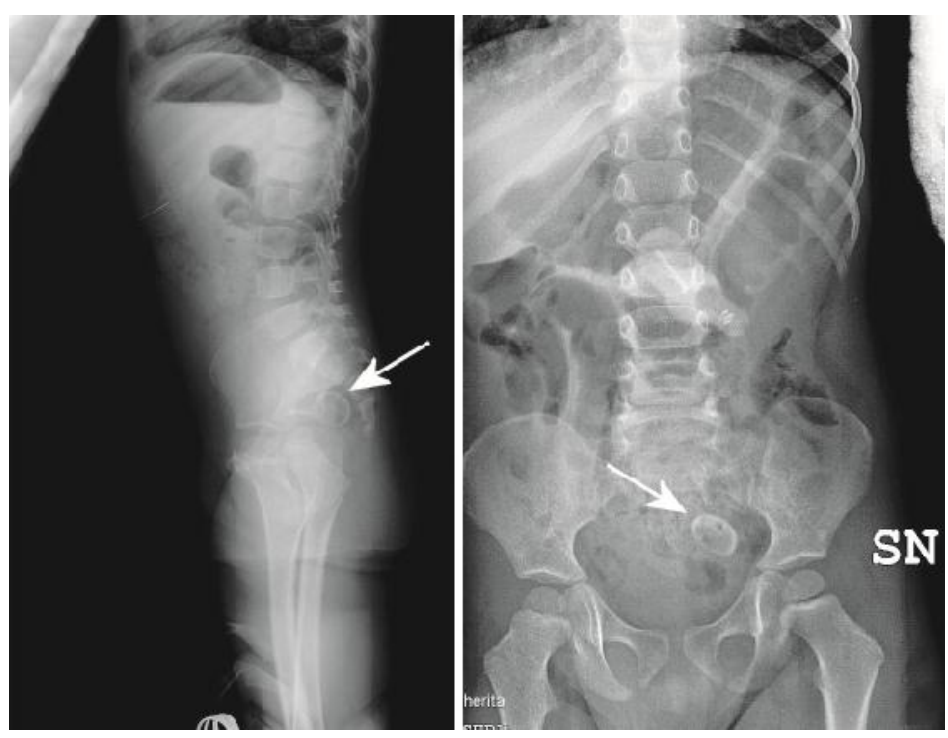

Fig. 1. Radiologic appearance of the bumper: abdominal X-rays showing the bumper localized in the left iliac fossa (arrows).

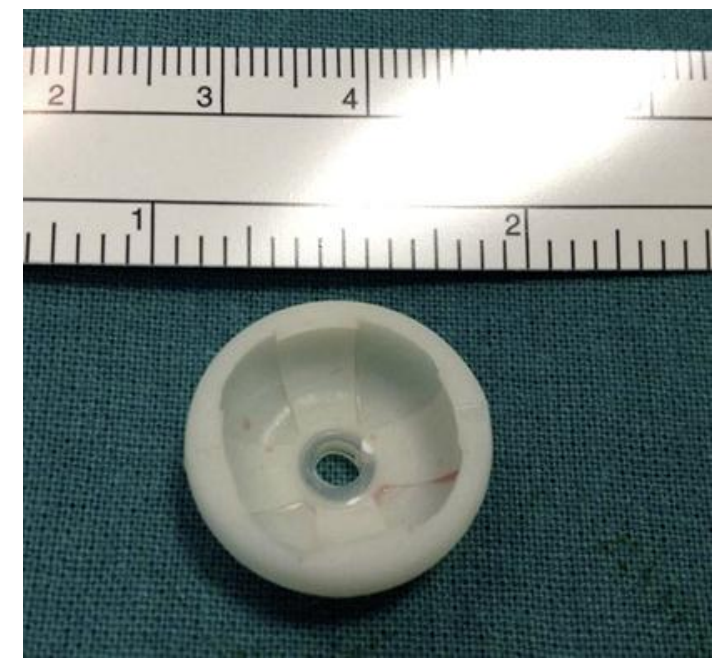

Fig. 2. Intraoperative findings: the bumper extracted laparoscopically from the umbilicus.

Guanà et al.: Gastrostomy Intraperitoneal Bumper Migration in a Three-Year-Old Child: A Rare Complication following Gastrostomy Tube Replacement

Fin. Intraoperative findings: the bumper extracted laparoscopically from the umbilicus. 